\title{
A Review About Pembrolizumab in First-Line Treatment of Advanced NSCLC: Focus on KEYNOTE Studies
}

This article was published in the following Dove Press journal: Cancer Management and Research

\section{Jialin $\mathrm{Qu}^{*}$ \\ Li Wang (D* \\ Man Jiang \\ Deze Zhao \\ Yuyang Wang \\ Feng Zhang \\ Jing Li \\ Xiaochun Zhang}

Department of Medical Oncology, The Affiliated Hospital of Qingdao University, Qingdao University, Qingdao 266003,

People's Republic of China

*These authors contributed equally to this work
Correspondence: Xiaochun Zhang Department of Medical Oncology, The Affiliated Hospital of Qingdao University, Qingdao University, 16 Jiangsu Road, Qingdao 266003, People's Republic of China

$\mathrm{Tel} / \mathrm{Fax}+865328291327$ I

Email zhangxiaochun9670@।26.com

\begin{abstract}
Lung cancer is currently the malignant tumor with the highest incidence and mortality in the world, while non-small cell lung cancer (NSCLC) is the most common pathological type of lung caner. In the past few decades, the only treatment options available for advanced NSCLC patients have been targeted therapy or chemotherapy, but these therapies are inevitably tolerated by tumors. The discovery of immune checkpints that mediate the immune escape of tumor cells have been promoting a series of immune checkpoint inhibitors to be used in cancer treatment and achieved great results. Among them, pembrolizumab is currently the only PD-1 inhibitor approved for first-line treatment of NSCLC, whether it is monotherapy or combination therapy, for creditable performance in KEYNOTE studies. In this review, we systematically integrate the latest series of clinical trial results, pharmacological mechanisms, adverse events (AEs) and predictive biomarkers in the first-line treatment of NSCLC. We hope pembrolizumab could become a better choice for more clinicians and benefit more patients with advanced NSCLC.
\end{abstract}

Keywords: pembrolizumab, PD-1/PD-L1, KEYNOTE studies, first-line treatment, nonsmall cell lung cancer, NSCLC

\section{Introduction}

Lung cancer is the malignant tumor with the highest incidence and mortality all over the world. 2,093,876 new cases of lung cancer were diagnosed and 1,761,007 patients died of lung cancer in 2018, accounting for $11.6 \%$ of the total number of diagnosed cancers and $18.4 \%$ of the total number of cancer deaths. ${ }^{1}$ Pathologically, lung cancer can be divided into two categories: small cell lung cancer (SCLC) and non-small cell lung cancer (NSCLC), the latter accounting for more than $85 \%$ of the total lung cancer. ${ }^{2}$ Traditional lung cancer treatment methods includes surgery, radiotherapy, chemotherapy and targeted therapy. However, only about $20 \%$ of patients with localdisease (stage I and II) have access to a radical resection. Thirty percent of patients with locally advanced disease and $50 \%$ of patients with metastatic disease are unable to completely clear the lesions via surgery. ${ }^{3}$ Targeted therapy seems to be the best option for these patients with inoperable advanced lung cancer that carry some driver gene mutation. ${ }^{4}$ But less than half of NSCLC patients have mutations in related tumor-driving genes including EGFR, BRAF, ERBB2 and rearrangement of ALK or ROS1, which can be blocked or inhibited by targeted medicine. ${ }^{5}$ For most patients with NSCLC that cannot be treated with targeted drugs, platinum-based 
chemotherapy has been used as a standard treatment for a long time, and the median survival time for these patients is about one year. ${ }^{6}$ Recently, it has been found that some molecules that can inhibit the function of immune cells was used by tumor cells to evade immune attack. We call the molecules with negative immune regulation immune checkpoints. ${ }^{7}$ As a key node in the process of tumorigenesis and development, immune checkpoints such as PD-1/PDL1, CTLA-4, TIM-3, LAG-3 and others provide us with a new therapeutic target. ${ }^{8}$ There has been particular focus on two immune checkpoint molecules, cytotoxic $\mathrm{T}$ lymphocyte antigen-4 (CTLA-4) and programmed cell death protein-1 (PD-1), which have been dedicated to have potent immune suppression effects through their function as negative regulators in the process of $\mathrm{T}$ cell activation. The available immune checkpoint inhibitors are usually monoclonal antibodies against these two molecules to restore the normal anti-tumor effect of $\mathrm{T}$ cells by blocking the interaction between these two molecules and their receptors or ligands. ${ }^{9}$ Immune checkpoint inhibitors have made breakthrough achievements in the field of tumor treatment and were verified in 12 types of malignancies (lung, melanoma, bladder, breast, colorectal, esophagus, gastric, head and neck, hematology, ovarian, pediatric, and other solid tumors). ${ }^{10}$

At present, the main checkpoint inhibitors in NSCLC are Pembrolizumab, Nivolumab and Atezolizumab. Among them, pembrolizumab is the only immune checkpoint inhibitor approved by the FDA and the National Medical Products Administration (NMPA) in China for first-line treatment of NSCLC. ${ }^{11}$

\section{Pharmacology Mechanism of Pembrolizumab}

Pembrolizumab approved by FDA to treat advanced NSCLC in 2015 is the first immune checkpoint inhibitor in NSCLC. ${ }^{12}$ The chemical nature of pembrolizumab is a humanized monoclonal antibody that specifically binds to PD-1 molecules on the surface of T cells. When pembrolizumab occupied the PD-1 precisely, steric hindrance effects can hinder the combination between PD-1 and its ligands, PD-L1 or PD-L2, to restore the normal anti-tumor immune response suppressed by PD-1 pathway. ${ }^{13}$

\section{PD-I and PD-LI/PD-L2}

PD-1 (CD279) was first discovered by Tasuku Honjo in 1992. PD-1 is a type I transmembrane protein that is expressed on the surface of activated T cells, B cells, monocytes and dendritic cells. The structure of PD-1 and CTLA-4 has 30\% homology, both belong to the CD28 molecular superfamily. ${ }^{14}$ The extracellular region of PD1 consists of a single IgV-like domain and the intracellular region retains an immunoreceptor tyrosine-based inhibitory motif (ITIM) and an immunoreceptor tyrosine-based switch motif (ITSM) depending on PD-1-mediated immune suppression. These regions were phosphorylated and recruit domain-containing protein-tyrosine phosphatase-2 (SHP-2) to inhibit the downstream signaling of T cell activation receptors after PD-1 binds its ligands. ${ }^{15}$

PD-L1 (B7-H1, CD274) and PD-L2 (B7-DC, CD273), two main ligands of PD-1, were isolated in 2009 and 2001 respectively, which is a remarkable achievement in profound understanding of physiological immune regulation. ${ }^{16}$ PD-L1 and PD-L2 belong to type I transmembrane glycoproteins and members of the B7 superfamily and they have a high degree of homology in sequence. ${ }^{17} \mathrm{PD}-\mathrm{L} 1$ is expressed on the surface of activated T cells, B cells, macrophages, and dendritic cells. In some cases, vascular endothelial cells, various interstitial cells, heart, lung, pancreas, and placental tissue can all be induced to express. PD-L1 restricts antigen-specific $\mathrm{T}$ cell responses and confines inflammatory tissue damage, which plays an important role in maintaining autoimmune tolerance and limiting the scale of inflammation. ${ }^{18}$ PD-L1 is also widely expressed in tumor tissues such as lung cancer, liver cancer, breast cancer, squamous cell carcinoma, and so on. It is expressed at low levels in normal tissues adjacent to the tumor, suggesting that it is involved in tumorigenesis and development and plays a key role in weakening the anti-tumor immune response. ${ }^{19}$

\section{Signal Pathway of PD-I/PD-LI}

PD-1/PD-L1 performs negative immune regulation mainly by inhibiting a series of T cell receptor (TCR) downstream signal transduction pathways, which play a center role to maintain self-tissue immune tolerance and keep immune balance physiologically. ${ }^{20}$ Changes in the PD-1/PD-L1 signaling pathway are closely related to the occurrence of many diseases, especially autoimmune diseases. However, tumor cells cleverly use this mechanism to escape immune killing. ${ }^{21}$ The transduction of PD-1/PDL1 signaling pathway requires not only the binding of PD1 to its ligand PD-L1, but also the interaction of major histocompatibility complex (MHC)-antigen peptide complex with TCR on the surface of T cells. When the 
combination between two receptors and ligands has reached the optimal state, it will lead to tyrosine phosphorylation of the PD-1 cytoplasmic region, thereby recruiting SHP-2 to the tyrosine of the PD-1 carboxyl-terminal ITSM motif. SHP-2 dephosphorylates TCR-related CD3z and ZAP70, thereby inhibiting multiple downstream signaling pathways, blocking cells in the G0/G1 phase and inhibiting $\mathrm{T}$ cell growth and cytokine release, which restrain $\mathrm{T}$ cells activation induced by TCR-CD3 complex. $^{22}$

\section{PLC-g/PIP2/Calcium Pathway}

When TCR recognizes tumor-associated antigens presented by MHC molecules expressed on the surface of tumor cells or antigen presenting cells (APC), T-cell activation signals are transduced from CD3 into cells. The $\mathrm{z}$ chain of $\mathrm{CD} 3$ molecule contains immunoreceptor tyrosine-based activation motif (ITAM) whose tyrosine residues can be phosphorylated after TCR binds to MHC molecules, recruiting tyrosine kinases with Src homology-2 (SH-2) domain such as ZAP-70 to activate. ${ }^{23}$ Then ZAP-70 phosphorylates tyrosine residues of the phospholipase C-g (PLC-g) to activate the PLC-g that splits phosphatidylinositol bisphosphate (PIP2) on cell membrane into two important secondary messengers: inositol 1,4,5-triphosphate (IP3) and diacyl glycerol (DAG). IP3 can open the calcium channels on the endoplasmic reticulum, increasing the calcium concentration in the cytoplasm by more than 10,000 times. High level of calcium can active calmodulin and calcineurin that dephosphorylate the nuclear factor of activated $\mathrm{T}$ cell (NF-AT) to make it to translocate from cytoplasm to nucleus. NF-AT and activating protein-1 (AP-1) form a complex that bind to the enhancer of interleukin-2 (IL-2) gene to initiate the transcription of IL-2 gene. IL-2 is the most important T cell autocrine growth factor that promote the division and differentiation of T cells. ${ }^{24} \mathrm{DAG}$ binds to free calcium ions in the cytoplasm to form complex that can phosphorylate the serine and threonine residues of protein kinase $\mathrm{C}(\mathrm{PKC})$ to activate it. Then activated $\mathrm{PKC}$ phosphorylate nuclear factor kappa-B (NF-kB) that transfer into nucleus, activating various cytokines gene transcription to amplify the immune response. ${ }^{25}$

PD-1 molecules are gradually expressed on the surface of T cells after T cells are activated by the downstream signaling pathway of the TCR-CD3 complex. ${ }^{26}$ The ITSM in C-terminal of PD-1 molecules is phosphorylated to recruit src homology 2-containing protein tyrosine phosphatase (SHP-2) when PD-1 bind to its ligand. SHP-2 can dephosphorylate TCR downstream signals CD3z and ZAP70 to inhibit the activation of NF-AT and NF-kB. So PD-1 can reduce release of IL-2 and other cytokines to hinder the proliferation and differentiation of $\mathrm{T}$ cells by inhibiting this pathways. $^{27}$

\section{PI3K/AKT Pathway}

The phosphorylation of ZAP70 results in rapid activation of phosphoinositide-3 kinase (PI3K) that promotes the generation of phosphatidylinositol 3,4,5-trisphosphate (PIP3). ${ }^{28}$ PIP3 binds downstream proteins AKT (protein kinase B, PKB) and phosphoinositide dependent kinase-1 (PDK1), causing PDK1 to phosphorylate the serine of AKT to activate it. AKT then phosphorylate and activate downstream signaling molecular mammalian target of rapamycin (mTOR). mTOR can directly phosphorylate a series of transcription factors to make related genes including cycle protein-dependent kinase (CDK) and pro-survival protein Bcl-XL, which help T cells proliferate and resist apoptosis. ${ }^{29}$

The dephosphorylation of ZAP70 induced by PD-1 decreases the expression of critical protein that promote T cell survival, causing T cell apoptosis. ${ }^{30}$ In addition, PD1 can further inhibit PI3K/AKT pathway by inducing expression of gene of phosphate and tension homology deleted on chromosome ten (PTEN). ${ }^{31}$

\section{Ras/MEK/ERK Pathway}

PD-1 also can inhibit MAPK cascade activated by TCRCD3 complex. When ZAP70 was activated, the linker for activating T cell (LAT) of ZAP70 can bind to the SH2 domain of growth factor receptor-bound protein 2 (Grb2) whose SH3 domain can collect son of sevenless (SOS) protein. SOS protein promote rat sarcoma (Ras) protein release GDP and bind to GTP, causing the molecular switch of the MAPK pathway to be opened. Then Ras protein hydrolyze the GTP to activate rapidly accelerated fibrosarcoma-1 (Raf-1) that can phosphorylate mitogenactivated protein kinase kinase (MEK-1/2). Extracellular regulated protein kinases (ERK) activated by MEK-1/2 is the last step in this cascade. ERK transfers from cytoplasm to nucleus and phosphorylates a series of transcription factors related to cell proliferation, providing growth signals to $\mathrm{T}$ cells. ${ }^{32,33}$ Dephosphorylating the ZAP-70 to reduce Ras/MEK/ERK pathway is a mechanism used by PD-1 to block the amplification of T cells. ${ }^{34}$

\section{Exosome PD-LI}

The traditional views suggest that PD-L1 is a membrane protein expressed on tumor cells and inhibits the function of T cells by binding to the PD- 1 on the surface of T cells. ${ }^{35}$ However, it is not so simple. Exosome that carry some 


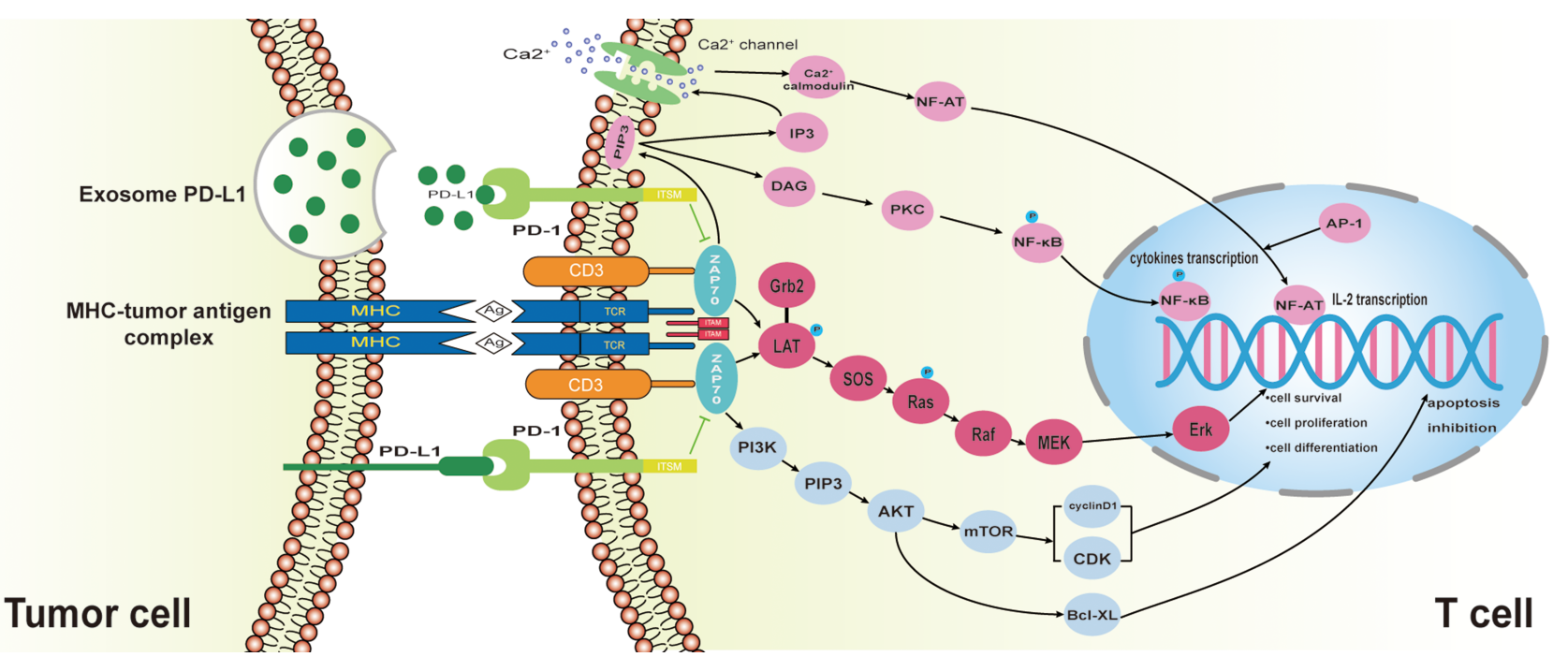

Figure I The Signal Transduction Pathway of PD-I / PD-LI. Under normal circumstances, MHC molecules on the surface of tumor cells present tumor-related antigens to TCR of T cells, which stimulates T cells to proliferate and differentiate into tumor-specific killer T cells through a series of downstream signaling pathways. But in the tumor microenvironment the combination of PD-I expressed on T cells with its ligand PD-LI expressed on tumor cells can inhibit the downstream signaling pathway of TCR, leading to $\mathrm{T}$ cell disability and tumor immune escape.

biomacromolecules such as complex RNA and proteins is a special form of extracellular vesicle (EV). ${ }^{36}$ Researchers have discovered that exosomes that carry a large number of PD-L1 are released by tumor cells and wander in the liquid phase, making immunosuppression mediated by PD-L1 is not limited to T cells that are directly in contact with tumor cells. In addition, exosomes in circulating blood can capture infused PD-1 antibodies like drones, which may be one of the reasons why some patients are ineffective for PD-1 antibody treatment. ${ }^{37}$ Taken together, these findings remind us that we should attach great importance to the role of PDL1 exosomes in tumor immune escape and that this mechanism may be a potential intervention target to understand and overcome the resistance of current immune checkpoint inhibitors (Figure 1).

\section{Pharmacokinetics and Pharmacological of Pembrolizumab} Pembrolizumab is a humanized monoclonal IgG4 antibody that is antagonistic toward PD-1. Binding of pembrolizumab to PD-1 cannot combine Fc receptors or activate complement, so that it is absence of any cytotoxic activity. ${ }^{38}$ It is a kind of lyophilized powder, dissolved in $0.9 \%$ sodium chloride solution before use to get the final concentration of $1-10 \mathrm{mg} / \mathrm{mL}$, which is used for intravenous infusion. Its chemical properties remain stable for up to four hours at room temperature and prolong to 24 hours in cold storage. In pharmacokinetic studies, pembrolizumab has displayed increased dose-proportional for peak and trough concentrations and the steady-state area-under-the-plasma concentration-time curves (AUC). The clearance increases with body weight gain. The dosage of pembrolizumab is 1$10 \mathrm{mg} / \mathrm{kg}$ every 2 weeks or $2-10 \mathrm{mg} / \mathrm{kg}$ every 3 weeks, based on this the clearance of pembrolizumab is $0.22 \mathrm{~L} /$ day with the serum half-life is $26 \mathrm{~d} .{ }^{39}$ Using a 3-weekly dosing regimen, steady-state concentration of pembrolizumab are reached by 18 weeks because clearance increases with body weight. AUC are $0.643-$ day/L and 3.77-day $/ \mathrm{mL}$ when we adopt an intravenous dose of $2 \mathrm{mg} / \mathrm{kg}$ and $10 \mathrm{mg} /$ $\mathrm{kg}$ every 3 weeks respectively. Renal function and mild hepatic impairment doses not affect the clearance rate of pembrolizumab. $^{40}$

FDA at April 28, 2020 has approved an additional recommended dosage of $400 \mathrm{mg}$ every six weeks (Q6W) for pembrolizumab across all adult indications, including monotherapy and combination therapy. This new dosage option will be available in addition to current dose of 200mg every 3weeks (Q3W). ${ }^{41}$

\section{Pembrolizumab as First-Line Treatment in NSCLC: Clinical Trials}

Treatments for advanced NSCLC without drive gene mutation have not progressed for decades before immune checkpoint inhibitors are put into clinical practice. ${ }^{42}$ In the past, the standard first-line treatment for advanced NSCLC patients without EGFR mutations or ALK/ROS1 rearrangements is platinum-double chemotherapy (PT-DC) whose objective response rate (ORR), the median progression- 
free survival (PFS) and overall survival (OS) were 15\%$32 \%, 4.0-5.1$ and $8.1-10.3$ months respectively. The OS rate of 1 - to 2-years were $30-44 \%$ and $10-18.9 \%$ respectively. ${ }^{43-45}$

Nowadays, pembrolizumab has made a revolutionary breakthrough in the treatment of advanced NSCLC, becoming the only immune checkpoint inhibitors for firstline treatment of advanced NSCLC. Through the results of a series of clinical trials of pembrolizumab, we take a look at the incomparable advantages of pembrolizumab as a first-line treatment option for NSCLC compared to traditional chemotherapy.

\section{First-Line Combined Therapy in Non-Squamous NSCLC}

Chemotherapy can promote immune activation through the following mechanisms to increase the anti-tumor activity of the immune system and the effect of immune checkpoint inhibitors. Chemotherapy causes a large number of tumor cell immunogenic deaths in a short time, which release tumor antigens and eliminate suppression of the immune system induced by tumor cells. Cytotoxic drugs on the one hand can directly enhance the function of activated $\mathrm{T}$ cells and $\mathrm{NK}$ cells, on the other hand can reduce the number of immunosuppressive cells, thereby indirectly enhancing the function of activated $\mathrm{T}$ cells. ${ }^{46}$ Among them, the immune activation effect of pemetrexed is very prominent. Pemetrexed induces the expression of most immune cell activation genes and positively regulates signaling pathways that play a key role in $\mathrm{T}$ cell recruitment or activation. ${ }^{47}$

Because of the synergy between chemotherapy and immune checkpoint inhibitors, the first-line combined treatment based on pembrolizumab and pemetrexed has achieved great success in non-squamous NSCLC.

In KEYNOTE-021G, a randomized, open-label, Phase 2 cohort of a multicohort study, 123 patients with chemotherapy-naïve, stage IIIB or IV, non-squamous NSCLC with negative EGFR or ALK genetic mutations were enrolled at 26 medical centers in the USA and Taiwan. These patients were randomly assigned to two groups: pembrolizumab plus chemotherapy group where 60 patients were treated with 4 cycles of pembrolizumab 200mg plus carboplatin area under curve $5 \mathrm{mg} / \mathrm{mL}$ per min and pemetrexed $500 \mathrm{mg} / \mathrm{m}^{2}$ every 3 weeks after pembrolizumab for 24 months, and the chemotherapy-alone group, where 63 patients were treated with unlimited pemetrexed maintenance therapy or 4 cycles of carboplatin and pemetrexed alone after unlimited pemetrexed maintenance therapy. As of the cutoff date of August 8,2016 , the median follow-up time had reached 10.6 months. The ORR of patients in the pembrolizumab plus chemotherapy group was $55 \%$, while only $29 \%$ in the chemotherapy alone group, which indicated that the combination between pembrolizumab and chemotherapy significantly increased the proportion of patients who achieved objective response. From the perspective of another secondary endpoint of this clinical trial, pembrolizumab plus chemotherapy significantly prolonged patients' PFS compared to chemotherapy alone. Median PFS had reached 13.0 months for pembrolizumab plus chemotherapy and only 8.9 months for chemotherapy alone. ${ }^{48}$ Based on 10.6 months follow-up data from the Phase II clinical trial KEYNOTE-021G, the FDA approved pembrolizumab plus carboplatin and pemetrexed as first-line treatment for metastatic non-squamous NSCLC without EGFR/ALK mutation on May 10, 2017. ${ }^{49}$

When the statistical data of KEYNOTE-021G were cut off on December 1, 2017, the median follow-up time for the two treatment groups was 23.9 months (range 0.8-35.1 months). The ORR was $56.7 \%$ with pembrolizumab plus chemotherapy versus $30.2 \%$ with chemotherapy alone. In terms of the main endpoint of KEYNOTE-021G, the ORR of pembrolizumab plus chemotherapy is about twice chemotherapy alone. Twenty-two of 60 patients in the pembrolizumab plus chemotherapy group (37\%) had died at the time of analysis, compared with 35 of 63 in the chemotherapy alone group (56\%). The median PFS is 24.0 months in patients in the pembrolizumab plus chemotherapy group, while 9.3 months in the chemotherapy alone group. Two-year follow-up results of KEYNOTE$021 \mathrm{G}$ confirm that pembrolizumab combined with chemotherapy for advanced non-squamous NSCLC reduced $47 \%$ risk of disease progression and $44 \%$ risk of death compared with traditional chemotherapy. On August 20, 2018, the 2-year follow-up results of KEYNOTE-021G were published in the Journal of Thoracic Oncology, which is the longest follow-up time for first-line immunotherapy combined with chemotherapy in NSCLC. ${ }^{50}$

Another clinical trial, KEYNOTE-189, further established the safety and antitumor activity of pembrolizumab combined with chemotherapy for first-line treatment of metastatic non-squamous NSCLC without EGFR/ALK mutation. In this randomized, multicenter, double-blind and placebocontrolled Phase III clinical trial, 616 patients with untreated metastatic non-squamous NSCLC without sensitizing EGFR/ALK mutation in a 2:1 ratio were randomly assigned 
to the pembrolizumab combination group (410 patients) where they received pemetrexed and carboplatin or cisplatin plus 200mg of pembrolizumab every 3 weeks for 4 cycles, followed by pembrolizumab for up to a total of 35 cycles plus pemetrexed maintenance therapy and placebo combination group (206 patients) where they were treated with the same procedures and medications, except that the pembrolizumab was replaced with placebo. In addition, more than one-third of patients in this trial had negative PD-L1 expression of tumor. Data analysis after a median follow-up of 10.5 months showed that pembrolizumab combined with chemotherapy reduced the risk of disease progression/death by nearly half. The OS rate at one year in the pembrolizumab combination group is estimated at $69.2 \%$ versus only $49 \%$ in the placebo combination group. Improvement in OS was observed across all categories classified by PD-L1 expression, which implied that patients can benefit from pembrolizumab combined with chemotherapy regardless of PD-L1 expression level. Furthermore, pembrolizumab also successfully improved PFS from 4.9 months in the placebo combination group to 8.8 months in the pembrolizumab combination group. From the perspective of the secondary endpoint of KEYNOTE189 , the ORR of pembrolizumab combined with chemotherapy was 2.5 times that of chemotherapy alone. The ORR in the pembrolizumab combination group was $47.6 \%$ compared with $18.9 \%$ in the placebo combination group. ${ }^{51}$ At the American Society for Clinical Oncology's 2020 Annual Meeting (ASCO), researchers announced the final analysis of KEYNOTE-189. The data cutoff date was May 20, 2019 (unpublished). The results showed that median OS in the ITT population was 22 months in the pembrolizumab combination group vs. 10.6 months in the placebo combination group; The two year survival rate was $45.7 \%$ vs. $27.3 \%$ and the HR was 0.56 . With long-term follow-up, pembrolizumab combined with chemotherapy continues to improve efficacy outcomes over placebo plus chemotherapy with median OS and PFS; approximately twice as long compared with placebo plus pemetrexed-platinum. Fifty-six patients received 35 cycles of pembrolizumab-combination therapy. Their ORR were $85.7 \%$ ( $4 \mathrm{CR}, 44 \mathrm{PR}, 8 \mathrm{SD}$ ). There were significant improvements of OS, PFS and ORR in patients received pembrolizumab combined with chemotherapy and no difference of efficacy between the carboplatin and cisplatin groups, which implied that the choice of chemotherapy drug has no effect on the survival benefit of patients. ${ }^{52}$ Due to the outstanding performance of pembrolizumab combined with chemotherapy in the treatment of advanced NSCLC demonstrated by KEYNOTE-021G and KEYNOTE-189, the NMPA in China published a list of 48 drugs needed urgently by clinics on August 8, 2018 where pembrolizumab is the only immunotherapy drug. ${ }^{53}$ Based on the KEYNOTE189 study, the FDA expanded the indications for pembrolizumab on August 20, 2018, approving pembrolizumab in combination with platinum-based drugs and pemetrexed for first-line treatment of metastatic non-squamous NSCLC. ${ }^{54}$

\section{First-Line Combined Therapy in Squamous NSCLC}

Great progress has been witnessed in the treatment of advanced NSCLC in this century, especially with the profound study of molecular events during tumorigenesis and development, more and more driver genes have been found in advanced lung adenocarcinoma to design molecular targeted drugs inhibiting precisely these driver genes, which have significantly prolonged the survival of patients with advanced lung adenocarcinoma. ${ }^{5-57}$ Lung squamous cell carcinoma accounts for about $15-20 \%$ of NSCLC. ${ }^{58}$ However, we still cannot know much about the molecular events of the emergence and development of lung squamous cell carcinoma. At present, the first-line treatment of advanced lung squamous cell carcinoma is still platinum-based chemotherapy. ${ }^{59}$ The five-year survival rate of patients with lung squamous cell carcinoma is only $5 \%$ after comprehensive treatment by surgery, radiotherapy and chemotherapy. ${ }^{60}$ KEYNOTE-024 study has confirmed the superiority of pembrolizumab compared to standard platinum-based chemotherapy for untreated patients with advanced NSCLC and high expression of PDL1 (PD-L1 TPS $>50 \%$ ). ${ }^{61}$ Based on these results, the 2017 version NCCN guidelines in oncology recommended that pembrolizumab was the first-line treatment for advanced NSCLC patients with PD-L1 TPS $>50 \%$ and negative mutations of EGFR/ALK regardless squamous cell carcinoma or non-squamous cell carcinoma. ${ }^{62}$ The study of KEYNOTE407 has received a positive result in the efficacy of pembrolizumab combined with chemotherapy as first-line treatment for advanced squamous NSCLC is presented in KEYNOTE-407.

In the KEYNOTE-407 study, there were 559 untreated patients with metastatic squamous NSCLC randomly assigned according to $1: 1$. The pembrolizumabcombination group patients received 4 cycles of pembrolizumab 200mg every 3 weeks plus carboplatin area under curve $6 \mathrm{mg} / \mathrm{mL}$ per min every 3 weeks and either paclitaxel $200 \mathrm{mg} /$ $\mathrm{m}^{2}$ every 3 weeks or nanoparticle albumin-bound-paclitaxel $100 \mathrm{mg} / \mathrm{m}^{2}$ every week followed by pembrolizumab $200 \mathrm{mg}$ 
every 3 weeks for 31 cycles and placebo-combination group patients received the same treatment plan except pembrolizumab replaced by placebo. This study is a double-blind, phase III trial. The statistical analysis at median follow-up of 7.8 months presented that the median OS of pembrolizumabcombination group was 15.9 months and placebocombination group 11.3 months. The OS benefit of patients was consistent whether their PD-L1 expression is high or not. The median PFS in pembrolizumab-combination group and placebo-combination group were 6.4 and 4.8 months. The result of KEYNOTE-407 trial has implied that the combination between pembrolizumab and chemotherapy consisted of carboplatin plus paclitaxel or nanoparticle albumin-boundpaclitaxel had led to surprisingly prolonged OS and PFS compared with chemotherapy alone. ${ }^{63}$ According to the significant achievement in KEYNOTE-407, pembrolizumab was approved by the NMPA in China for first-line treatment of metastatic squamous NSCLC in combination with chemotherapy, which is the third approval for pembrolizumab in first-line NSCLC in China in less than one year. ${ }^{64}$

\section{First-Line Monotherapy in NSCLC}

Although chemotherapy can elevate the effect of immune checkpoint inhibitors to varying degrees, the cytotoxic drugs used in standard chemotherapy have inherent defects: more adverse reactions. Some older or poorer patients may not be able to tolerate the side effects of chemotherapy, so immunotherapy alone appears to be the only option. Studies have shown that immune checkpoint inhibitor single-agent treatment has a lower incidence of adverse reactions and mortality than standard chemotherapy on the premise of ensuring long-term survival of patients. ${ }^{65}$ In some patients with high PD-L1 expression, the growth and progression of tumor cells are dependent on the immune escape mediated by the PD-1/PD-L1 pathway excessively. Therefore monotherapy with immune checkpoint inhibitors can also achieve great therapeutic effects in these patients, which has been verified in KEYNOTE-024 and KEYNOTE-042 trials. ${ }^{66,67}$

KEYNOTE-024 is the first clinical trial to use pembrolizumab in first-line treatment for advanced NSCLC. This international, randomized, open-label, phase III study was designed to compare the advantage of pembrolizumab monotherapy and platinum-based chemotherapy. Three hundred and five patients suffered from untreated advanced NSCLC with PD-L1 tumor proportion score (TPS) of $50 \%$ or greater and without EGFR/ALK mutations in 1:1 ratio were randomly assigned to pembrolizumab group and platinum-based chemotherapy group. One hundred and fifty four patients in pembrolizumab group have received $200 \mathrm{mg}$ intravenous pembrolizumab every 3 weeks for 35 cycles or 2 years. Another 151 patients first have received PT-DC for 4 to 6 cycles and they were cross-treated with pembrolizumab every 3 weeks for 2 years if they had a progressive disease (PD). At the median follow-up time of 11.2 months, median PFS has reached 10.3 months in the pembrolizumab group compared with 6.0 months in the chemotherapy group and OS at 12 months for pembrolizumab and chemotherapy were estimated to $70.3 \%$ and $54.8 \%$ by Kaplan-Meier survival analysis, which dedicated that pembrolizumab reduced the risk of disease progression or death by half versus chemotherapy. In addition, the ORR were $44.8 \%$ and $27.8 \%$ in pembrolizumab group and pembrolizumab has a lower incidence of treatment-related adverse events (TRAEs) of any grade compared to chemotherapy ( $73.4 \%$ vs. $90.0 \%)$, as has grade 3 , 4 , or $5(26.6 \%$ vs. $53.3 \%) .{ }^{61}$ Based on the previous finding of KEYNOTE-024, the FDA approved pembrolizumab as a first-line treatment for metastatic NSCLC with PDL1 expression of $50 \%$ or greater and without EGFR/ALK mutations on October $24,2016 .{ }^{68}$ At the cutoff date of July 10, 2017, the median follow-up time has reached 25.2 months. During this period, the median OS of the pembrolizumab group was 30.0 months and the chemotherapy group was 14.2 months, which illustrated that pembrolizumab reduced the risk of death by $37 \%$ compared to chemotherapy and continued to verify that pembrolizumab significantly increased the OS of patients. ${ }^{67}$ This trial outcome is more advantaged than that presented in trials assessing platinumbased chemotherapy as first-line treatment of NSCLC. ${ }^{69}$ Nivolumab, the equal of pembrolizumab, did not achieve significant survival benefits as first-line treatment in patients with advanced NSCLC in CheckMate-026 study. ${ }^{70}$ So KEYNOTE-024 is the first clinical trial to display benefit of OS with anti-PD-1 monotherapy versus traditional chemotherapy as first-line treatment in advanced NSCLC and pembrolizumab have been the only PD-1 inhibitors for firstline monotherapy in NSCLC that has changed the treatment paradigm of the disease.

A series of clinical trials of pembrolizumab in first-line NSCLC treatment showed that merely $30 \%$ of patients receiving pembrolizumab belonged to PD-L1 TPS of $50 \%$ or greater without EGFR/ALK gene mutations. ${ }^{51,71}$ A real-world study aimed to explore the PD-L1 expression in patients with locally advanced or metastatic NSCLC dedicated that PD-L1 TPS $\geq$ $50 \%, \geq 1 \%$, and $<1 \%$ accounted for $22 \%, 52 \%$, and $48 \%$, respectively, in the 2368 qualified tumor samples. ${ }^{72}$ The 
purpose of this is to prove whether patients with PD-L1 expression in $1-49 \%$ also benefit from pembrolizumab monotherapy. KEYNOTE-042 is a randomized, open-label, controlled, phase III trial to investigate the OS of pembrolizumab monotherapy in NSCLC patients with PDL1 expression of $1 \%$ or greater.

One thousand two hundred and seventy-four patients with PD-L1 expression $\geq 1 \%$ in a $1: 1$ ratio were randomly allocated to the pembrolizumab group $(n=637)$ and the chemotherapy group $(n=637)$. At the same time, the pembrolizumab group and chemotherapy group were divided into 3 subgroups according to the PD-L1 TPS level of patients: TPS $1-19 \%$, TPS $20-49 \%$, and TPS $\geq 50 \%$. Patients in the pembrolizumab group were treated intravenously with pembrolizumab $200 \mathrm{mg}$ alone every 3 weeks for up to 35 cycles, while the chemotherapy group received carboplatin to achieve an area under the curve of $5-6 \mathrm{mg} / \mathrm{mL}$ per min plus paclitaxel $200 \mathrm{mg} / \mathrm{m}^{2}$ or pemetrexed $500 \mathrm{mg} / \mathrm{m}^{2}$ every 3 weeks for 4 or 6 cycles followed by optional pemetrexed $500 \mathrm{mg} / \mathrm{m}^{2}$ every 3 weeks for maintenance therapy. In this study, crossover from the chemotherapy group to pembrolizumab was unallowed. When the median follow-up time had reached 12.8 months at Feb 26, 2018, the significantly longer OS was observed in pembrolizumab group than in chemotherapy group in all 3 TPS subgroups. The median survival times in 3 TPS subgroups were 16.7 months for pembrolizumab compared with 12.1 months for chemotherapy, 17.7 months compared with 13.0 months and 20.0 months compared with 12.2 months, respectively. Pembrolizumab also showed unparalleled advantages in terms of AEs. The incidence of treatment-related AEs in the pembrolizumab group was $63 \%$ (399), much lower than the 90\% (553) in the chemotherapy group. The statistical data of KEYNOTE-042 that choose OS to be the primary endpoint confirm the reason of pembrolizumab monotherapy as a first-line treatment for NSCLC, regardless of whether PD-L1 expression is high or low. ${ }^{66}$ Based on the results of KEYNOTE-042, FDA broadened the indications for pembrolizumab on April 11, 2019, approving pembrolizumab for the firstline treatment of patients with stage III NSCLC who are not candidates for surgical resection or definitive chemoradiation or metastatic NSCLC with PD-L1 TPS $\geq 1 \%$ and without EGFR or ALK genomic aberrations. ${ }^{73}$ Table 1.

\section{Adverse Events of Pembrolizumab}

Many patients cannot tolerate the toxic side effects of antitumor drugs, which is one of the important reasons for treatment interruption, or even death, of cancer patients. ${ }^{74}$ Multiple clinical trials have shown that the incidence rate of TRAE with pembrolizumab monotherapy is much lower than conventional chemotherapy (76.6\% vs. $90 \%$ in KEYNOTE024 and $63 \%$ vs. $.90 \%$ in KEYNOTE-042) and with pembrolizumab, combined chemotherapy is similar to conventional chemotherapy (99.8\% vs. 99\% in KEYNOTE-189). ${ }^{52,66,67}$ But as a PD-1 inhibitor, pembrolizumab has unique adverse reactions. PD-1 inhibitors can block the negative regulatory signals of $\mathrm{T}$ cells to terminate immune suppression and enhance the anti-tumor effect of T cells. However, this mechanism may also excessively activate $\mathrm{T}$ cells, which leads to imbalance of immune tolerance and performs autoimmune-like inflammatory response called immune-related adverse events (irAEs) when it affects normal tissues including skin, digestive tract, liver, endocrine gland, lungs, and so on. ${ }^{75}$ A meta-analysis has pointed that serious organ specific irAEs were rare, but rates of these events were increased with pembrolizumab compared with standard treatment. ${ }^{76}$ Therefore, we reviewed the irAEs of various organs caused by the application of pembrolizumab to help early diagnosis and intervention.

\section{Skin Toxicity}

The most common and earliest occurrence of irAEs is skin toxicity, including follicular dermatitis, erythematous dermatitis, and popular dermatitis. ${ }^{77}$ The peak period occurs after two cycles of pembrolizumab and mild skin toxicity is the most common, about $10 \%$ of patients experience varying degrees of itching, rashes, and eczema. ${ }^{78}$ The literature reports that lichen-like skin reactions and eczema can evolve into vitiligo whose mechanism may be that immune checkpoint inhibitors blocked certain antigens expressed in tumors and skin. ${ }^{79}$ In addition, PD-1 blockers increase the sensitivity of radiotherapy, so the combination of the two treatments may induce acute skin reactions. ${ }^{80}$ In addition, a study of 83 patients, including 15 patients with lung cancer, treated with pembrolizumab, showed that patients with cutaneous AEs had significantly longer PFS. Especially the hypopigmentation in melanoma patients might represent a better prognosis, which may be derived from common antigen between melanocytes and melanoma and cross-reaction. Therefore, the appearance and development of cutaneous AEs may be a positive prognostic sign for tumor patients receiving pembrolizumab. ${ }^{81}$

\section{Gastrointestinal Reaction}

Gastrointestinal reaction mostly occurred 5 to 10 weeks after the application of pembrolizumab. The clinical manifestations are mainly diarrhea and colitis. ${ }^{82}$ The incidence 


\begin{tabular}{|c|c|c|c|c|c|}
\hline 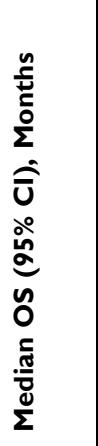 & 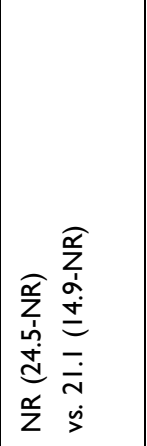 & 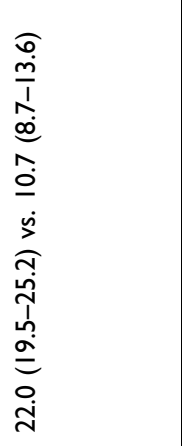 & 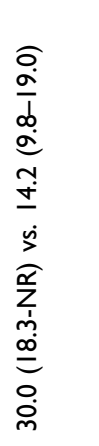 & 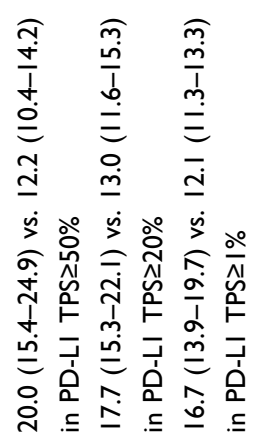 & 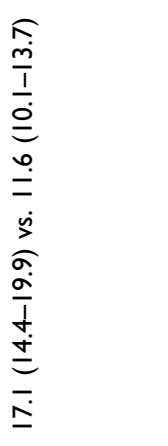 \\
\hline 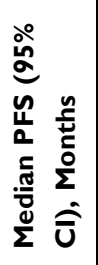 & 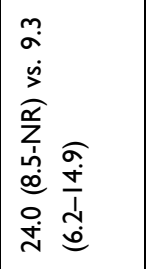 & 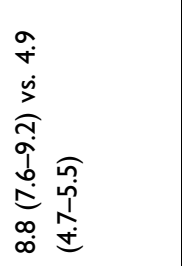 & 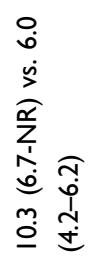 & 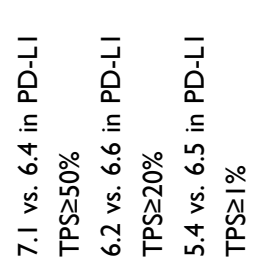 & 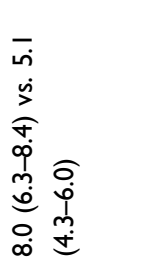 \\
\hline 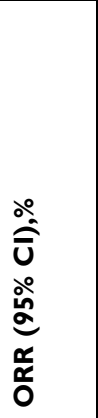 & 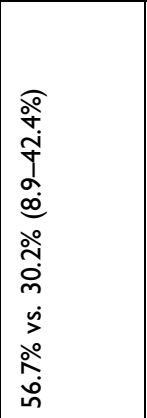 & 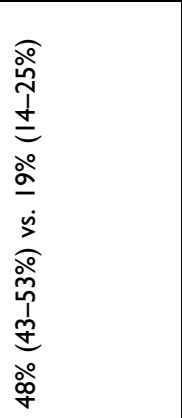 & 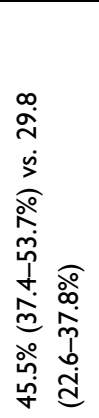 & 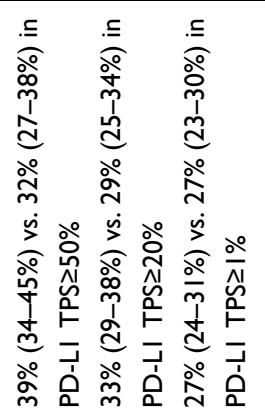 & $\begin{array}{l}\stackrel{\circ}{0} \\
\infty \\
m \\
\dot{s} \\
\dot{\circ} \\
\stackrel{\circ}{0} \\
\text { j. }\end{array}$ \\
\hline 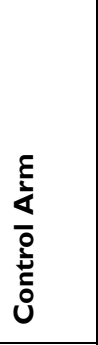 & 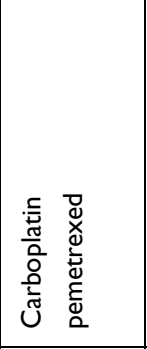 & 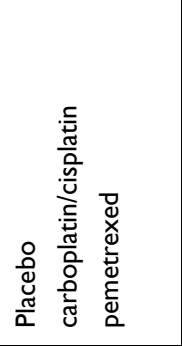 & 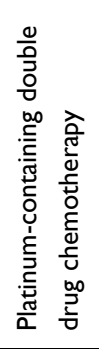 & 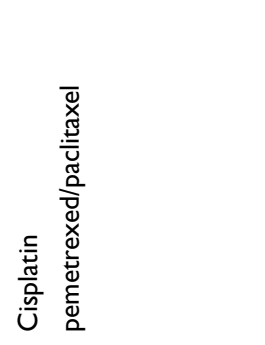 & 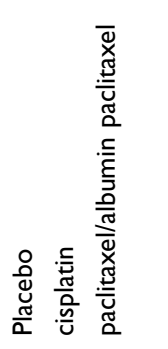 \\
\hline 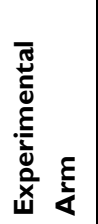 & 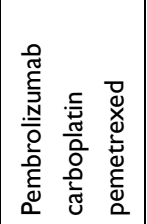 & 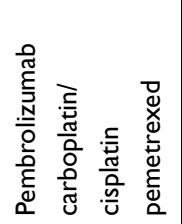 & 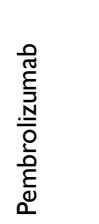 & 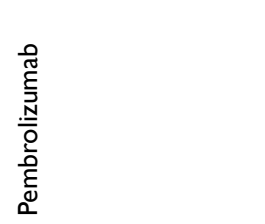 & 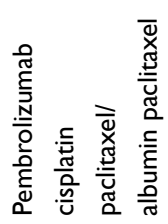 \\
\hline$=$ & $\stackrel{\tilde{a}}{\underline{a}}$ & 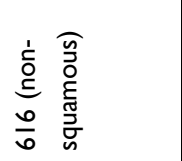 & 岏 & $\stackrel{+}{\text { I }}$ & 施 \\
\hline 㞼 & 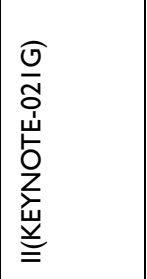 & 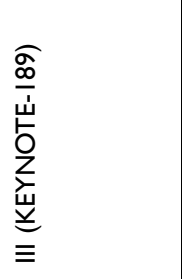 & 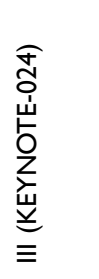 & 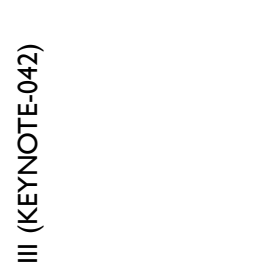 & 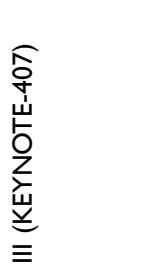 \\
\hline
\end{tabular}


of diarrhea is 5- $10 \%$, which uis usually mild or moderate. ${ }^{83}$ Colitis refers to diarrhea accompanied by any of the following symptoms: abdominal pain, rectal bleeding and increased mucus secretion or pathological examination under endoscopy showing changes of colitis. The rate of immune-related colitis is $1.3 \%$, which increase to $11.8 \%$ when patients receive combination treatment. ${ }^{84}$

\section{Autoimmune Liver Disease}

The liver damage caused by pembrolizumab are usually immune-mediated hepatitis with incidence of less than 5\%, which most often occur after 8 to 13 weeks of medication. ${ }^{40}$ It is reported that liver damage can be delayed until several years after taking the medicine, so doctors should pay attention to it during follow-up. ${ }^{85}$ The symptoms of pembrolizumab-related hepatotoxicity can manifest as jaundice, epigastric pain, vomiting, as well as asymptomatic or other non-specific symptoms such as fever, fatigue, indigestion, and so on. ${ }^{86}$ The imaging finding of irAEs with liver damage are not typical. In severe cases, CT scans show mild hepatomegaly and enlarged lymph nodes around the portal vein. ${ }^{87}$

Pathological manifestations in the liver were edema in the manifold area, infiltration of lymphocytes inside the liver sinus, and the bile duct orifice. ${ }^{88}$

\section{Immunotherapy-Associated Pneumonitis}

Immunotherapy-associated pneumonia caused by PD-1/ PD-L1 blockers is mainly manifested as non-specific interstitial pneumonia, which usually occurs anytime between 2 weeks and 2 years ${ }^{89}$ This reaction occurs earlier in lung cancer patients than in other tumor patients, which may be related to the heavy tumor burden in the lung. ${ }^{90}$ Once using pembrolizumab, immunotherapy-associated pneumonia develops in 3-8\% of patients and in a few patients it can be life-threatening. ${ }^{91}$ It has also been reported that patients with lung cancer who have a previous history of emphysema, asthma, pulmonary fibrosis, and lung radiotherapy may have greater and more severe immune-related pneumonia. ${ }^{92}$

The symptoms of irAEs pneumonia include dry cough, progressive dyspnea, fever and chest pain. Patients with suspected irAEs pneumonia require a CT scan of the lung, whose most common imaging manifestation is organizing pneumonia such as ground-glass changes, grid-like changes, and pulmonary consolidation. Multiple lesions mostly locate in the double lower lungs. ${ }^{93}$

\section{Endocrine Organ Damage}

Among patients treated with pembrolizumab, about $10 \%$ were observed to have different levels of immune-related endocrine disorders where more common are thyroid dysfunction and pituitary inflammation, and type I diabetes has also been reported. ${ }^{66}$ Pembrolizumab-mediated endocrine toxicity mainly affects the thyroid gland, leading to grade 1 or 2 hypothyroidism (4-8\%), hyperthyroidism (2-5\%), and rare acute thyroiditis $(<1 \%) .{ }^{39}$ If there are decrease or increase in TSH and previous thyroid dysfunction in clinical, free triiodothyronine (fT3) and free thyroxine (fT4) should be monitored during pembrolizumab application. ${ }^{94}$

The incidence of pituitary inflammation ranges from $1 \%$ to $6 \%$, including hypopituitarism and adrenal insufficiency. Symptoms of pituitary inflammation can be ambiguous with mild fatigue, joint pain, behavioral changes and loss of sexual desire due to insufficient hormones even severe headache and visual changes, which are often atypical and difficult to diagnose. ${ }^{95}$ Therefore regular monitoring of TSH, fT3 and fT4 is necessary, as low TSH may be a precursor to pituitary inflammation. ${ }^{96}$

Recently, it has been reported that PD-1 antibody can induce type I diabetes. A cancer patient without any personal or family history of diabetes developed autoimmune type I diabetes and diabetic ketoacidosis after 7 weeks of treatment with PD-1 antibody. Type I diabetes, a severe insulin deficiency, is extremely susceptible to fluctuations in blood sugar and can be life-threatening. Once diagnosed, PD-1 antibody use should be discontinued immediately and insulin replacement therapy should be initiated. ${ }^{97}$ KEYNOTE's series of clinical trials have also seen several patients who suffered from type I diabetes after using pembrolizumab. ${ }^{78,86,91}$ Although the incidence is low, doctors should be more vigilant due to the potential danger.

The incidence of irAEs is high but most patients have mild events and a better prognosis. The key point is early detection and intervention. Mild patients merely need symptomatic treatment without interrupting immunotherapy. If the disease has been developing progressively, immunotherapy needs to be suspended and glucocorticoids given as appropriate. For patients with severe adverse reactions, immunotherapy must to be stopped permanently and high-dose glucocorticoids should be combined with immunosuppressants such as cyclophosphamide, cyclosporine $\mathrm{A}$ and infliximab and so on. In addition, damage of endocrine organs requires temporary or permanent hormone replacement therapy. ${ }^{98-101}$ 


\section{Predictive Biomarkers of Pembrolizumab}

Pembrolizumab has provided a new first-line option for patients with advanced NSCLC and patients can achieve sustained remission once they respond to it, but less than $30 \%$ of patients will respond to pembrolizumab. ${ }^{102,103}$ There is an urgent need for relevant biomarkers to screen those who had an effective response to pembrolizumab, so that we can accurately predict the efficacy and prognosis of pembrolizumab, bring long-term and safe survival benefits, and use the limited medical and economic resources as efficiently as possible.

\section{Biomarkers to Screen Dominant Populations PD-LI Expression}

Overexpression of PD-L1 in tumor cells can participate in the immune escape of tumors by inhibiting the function of cytotoxic T cells. ${ }^{104}$ Therefore, PD-L1 expression is considered as a biomarker to predict the efficacy of pembrolizumab. The immunohistochemistry (IHC) to detect PD-L1 expression from tumor tissues is one of the commonly used methods to predict the effectiveness of PD-1/PD-L1 antibody in clinic. ${ }^{105}$

In the clinical trial of PD-1 antibody second-line monotherapy for NSCLC, Checkmate-057 of nivolumab and KEYNOTE-010 of pembrolizumab, the expression of PDL1 was positively correlated with the efficacy of immunotherapy. ${ }^{86,106}$ In KEYNOTE-042, a first-line monotherapy of pembrolizumab for NSCLC, the median OS of 3 subgroups according to PD-L1 expression (PD-L1 TPS $\geq 1 \%, \geq 20 \%$ and $\geq 50 \%$ ) increased in order $(16.7,17.7$ and 20.0 months). ${ }^{66}$ When pembrolizumab combined with chemotherapy is used as first-line treatment for NSCLC, PD-L1 expression has little correlation with efficacy. The results of KEYNOTE-189 and KEYNOTE-407 showed that OS has significant benefits regardless of PD-L1 expression. ${ }^{107,108}$ However, other studies have put forward different opinions that NSCLC patients with low or no expression of PD-L1 can also benefit from anti-PD-1 treatment. ${ }^{109}$ A similar phenomenon also occurs in other tumor types such as gastric cancer, renal cell carcinoma and urothelial carcinoma, which indicates that PD-L1 expression alone cannot accurately predict the efficacy of immune checkpoint inhibitors. ${ }^{110-112}$

\section{Tumor Mutation Burden}

Tumor Mutation Burden (TMB) provides a quantitative estimate of the total number of mutations in the coding region of the tumor genome, so tumor cells with high levels of TMB may be more easily recognized by the immune system, which in turn triggers a stronger immune response to immune checkpoint inhibitors. ${ }^{113}$ Multiple studies have shown that TMB is associated with the efficacy of immune checkpoint inhibitors. NSCLC patients with a high non-synonymous mutation load displayed more sustained clinical benefit in the treatment of pembrolizumab. ${ }^{114}$ NSCLC patients who lack the mutation-associated neoantigen may develop acquired resistance during the treatment of immune checkpoint inhibitors. ${ }^{115}$ This phenomenon also exists in tumors of the urinary system. Rosenberg et al have found that tumor molecular subtypes and TMB are two independent factors predicting the response of atezolizumab in the treatment of bladder cancer. ${ }^{116}$ These results have shown that TMB is expected to be next effective biomarker to predict the efficacy of immune checkpoint inhibitors.

\section{MMR/MSI}

DNA mismatch repair (MMR) is an important DNA damage repair mechanism that recognizes and repairs bases that have mismatches, deletions and insertions during DNA replication and recombination. ${ }^{117}$ In a variety of tumors, mismatch repair deficiency (dMMR) can cause TMB to multiply, increasing patient reactivity to immune checkpoint inhibitors. $^{118}$ High level of microsatellite instability (MSI-H) is an important feature of dMMR tumors and MSI-H or MMR protein deletions are often used to identify this genotype. ${ }^{119}$ The KEYNOTE-016 clinical study has analyzed 149 tumor patients with MSI$\mathrm{H}$ or dMMR characteristics. The ORR of these patients treated with pembrolizumab was $39.6 \%$, of which 11 patients $(7.4 \%)$ had a complete response and 48 patients $(32.3 \%)$ had a partial response. Of the patients who responded effectively to pembrolizumab, 78\% had a response time of at least 6 months. ${ }^{120}$ Based on five nonblind, multi-group phase I/II clinical trials, the FDA accelerated approval of pembrolizumab for patients who have metastatic or unresectable solid tumors with MSI-H or dMMR. ${ }^{121}$ Pembrolizumab is the first anti-cancer drug that does not differentiate based on tumor location or source but specific genetic background and MSI-H or $\mathrm{dMMR}$ is also the only recognized predictive biomarker of the efficacy of immune checkpoint inhibitors. However, in advanced solid tumors, MSI-H patients are only $4 \%$ to $5 \%$ and the response rate of these patients to immune checkpoint inhibitors is only about $40 \% .^{122}$ Therefore, 
other new immunotherapy biomarkers are urgently needed to guide the treatment in clinical practice.

\section{Biomarkers to Screen Ineffective and Hyperprogression Populations}

A phase II clinical study, NCT02879994, was designed to explore the efficacy and safety of pembrolizumab in advanced NSCLC patients with EGFR-positive, PD-L1positive (PD-L1 TPS $\geq 50 \%$ ) and tyrosine kinase inhibitors (TKI) naive. The trial planned to enroll 25 patients but was terminated early due to lack of efficacy when enrolled to 11. The conclusion of the study indicated that there is a lack of efficacy in advanced NSCLC patients with EGFR-positive and TKI naive including those with PDL1 TPS $\geq 50 \%$, which may mean that immune checkpoint inhibitors is not suitable for such patients. ${ }^{123}$

Hyperprogression after immunotherapy refers to the explosive progression of tumors after the application of immune checkpoint inhibitors whose incidence is 9-29\%. After the patients have hyperprogression, the prognosis is often extremely poor and the median survival time is only 2 to 5 months. ${ }^{124}$ Kato et al further explored genetic markers that may be related to hyperprogression after immunotherapy. The results showed that MDM2/MDM4 amplification and EGFR mutations were connected with the hyperprogression after treatment with PD-1/PD-L1 inhibitors but the specific mechanisms are not clear. ${ }^{125}$

\section{Discussion}

Nowadays, immune checkpoint inhibitors represented by pembrolizumab have achieved brilliant improvement in the field of tumor therapy including NSCLC, opening a brand-new model in tumor therapy: immune normalization. ${ }^{126}$ But behind the remarkable achievements, there are still shortcoming in pembrolizumab that we need to discuss and address urgently.

First of all, can the treatment time window of pembrolizumab be moved forward again? Pembrolizumab was initially used as a second-line or even third-line treatment for advanced NSCLC. Due to tumor burden overload, chronic malnutrition and lymphocyte depletion, many patients with advanced NSCLC have missed the best time to treat pembrolizumab when they received secondline immunotherapy. According to the results of a series of KEYNOTE studies, it is now the consensus that pembrolizumab is suitable for first-line treatment of patients with unresectable advanced NSCLC. But the best time to cure cancer is in the early stages, so the feasibility of pembrolizumab for patients with early NSCLC that can be surgically removed is worth exploring. Preliminary results of a new adjuvant treatment for nivolumab in surgically resectable NSCLCs have indicated that patients receiving new adjuvant immunotherapy have varying degrees of pathological remission and their relapse-free survival (RFS) rate at 18 months has reached $73 \%{ }^{127}$ At the same time, clinical trials of pembrolizumab in new adjuvant therapy for early breast cancer are ongoing. ${ }^{128}$ Implementing new adjuvant immunotherapy in earlystage cancer patients is an attractive idea because the primary tumor provides the immune system with a wealth of tumor-specific antigens and the immune status of patients is better. We also hope that the clinical trials of new adjuvant therapy for pembrolizumab in early-stage NSCLC can be started as soon as possible, which is a potential milestone because it may have important implications for the development of new adjuvant treatment strategies in NSCLC.

Secondly, the deficiency of reliable biomarkers to accurately predict efficacy of pembrolizumab is another tricky problem. PD-L1 expression that is necessary before the medication of pembrolizumab had been verified to be an unsatisfactory biomarker. Factors such as intertumoral and intratumoral heterogeneity, interventions, cell types and staining sites can affect PD-L1 expression. ${ }^{129} \mathrm{TMB}$ may be an excellent biomarker for predicting the effectiveness of immune checkpoint inhibitors in NSCLC. But there are still many problems with TMB as a predictive biomarker. For example, the current cost of TMB detection is still expensive because it relies on gene sequencing, inconsistent testing standards of TMB across different platforms prevents physicians from accurately judging and the level of TMB is not exactly consistent with the response to immune checkpoint inhibitors. For MSI-H, the appearance of this phenotype is highly correlated with tumor type. Tumor types with MSI-H are usually digestive tract tumors and endometrial cancers but in NSCLC, MSI-H is uncommon. ${ }^{130}$ In addition, the current PD-L1, TMB and MSI- $H$ test are mainly based on tumor tissue. However, many patients cannot have enough tumor tissue to use and even some patients are not suitable for tissue biopsy. In NSCLC, nearly $30 \%$ of patients have this condition. ${ }^{131}$ These issues prevent us from effectively screening the right population for pembrolizumab, wasting limited medical and economic resources. Fortunately, with the development of emerging 
biomarkers such as intestinal microbiota and exosomes of PD-L1, the combined detection of multiple biomarkers will make immunotherapy more and more accurate. In addition, dynamic changes of each biomarkers should be analyzed carefully by physicians during treatment process, which is very important to adjust the individualized treatment plan.

Finally, cancer treatment is a systematic project dependent on the cooperation of various treatment methods. Pembrolizumab, on behalf of immune checkpoint inhibitors, can be combined with classical treatments such as surgery, chemotherapy, radiotherapy, targeted therapy and immunotherapy to achieve cumulative or synergistic therapeutic effects. Surgery, chemotherapy and radiotherapy can destroy tumor cell to release tumor antigens. Targeted therapy can reshape the tumor's immune microenvironment, increase the number of infiltrating lymphocytes and induce the expression of PD-L1 to enhance the efficacy of immune checkpoint inhibitors. ${ }^{132}$ PD-1 antibody and CTLA-4 antibody have also been successfully used in NSCLC. ${ }^{133}$ We can expand treatment methods to enhance the antitumor effect, but the best drug combination, sequence and timing should be determined after accumulating more data and experience from the clinic.

\section{Conclusion}

Currently, immune checkpoint inhibitors represented by pembrolizumab have revolutionized the field of treatment in advanced NSCLC. The achievement that pembrolizumab can induce durable tumor regression with few AEs by restoring the normal anti-tumor ability of immune system has been witnessed in KEYNOTE studies. On the basis of KEYNOTE clinical trials, more clinical research and basic experiments are also ongoing to find better combined treatment options, explore new biomarkers and develop a new generation of immune checkpoint inhibitors. With the continuous enrichment of clinical data and profound explore of specific mechanisms, pembrolizumab will definitely transform advanced lung cancer from a highmortality malignant disease to a controllable chronic disease like hypertension and diabetes.

\section{Highlights}

- Immune checkpoint inhibitors are an emerging treatment for advanced NSCLC, which may induce longterm tumor regression with minimal side effects.

- Pembrolizumab is currently the only PD-1 inhibitor approved for first-line treatment of advanced NSCLC.
- In KEYNOTE clinical trials, pembrolizumab has achieved remarkable results in first-line treatment of patients with advanced NSCLC, either alone monotherapy or combined therapy.

\section{Acknowledgments}

This work was supported by the Taishan Scholar foundation (NO tshw201502061). We thank Li lin from MSD China Medical Affair for manuscript revision and help on proofreading.

\section{Disclosure}

The authors report no conflicts of interest in this work.

\section{References}

1. Bray F, Ferlay J, Soerjomataram I, et al. Global cancer statistics 2018: GLOBOCAN estimates of incidence and mortality worldwide for 36 cancers in 185 countries. CA Cancer J Clin. 2018;68 (6):394-424. doi:10.3322/caac.21492

2. Siegel RL, Miller KD, Jemal A. Cancer statistics, 2018. $C A$ Cancer J Clin. 2018;68(1):7. doi:10.3322/caac.21442

3. Chansky K, Detterbeck FC, Nicholson AG, et al. The IASLC lung cancer staging project: external validation of the revision of the TNM stage groupings in the eighth edition of the TNM classification of lung cancer. J Thorac Oncol. 2017:S1556086417303404.

4. Kwak EL, Bang Y-J, Camidge DR, et al. Anaplastic lymphoma kinase inhibition in non-small-cell lung cancer. $N$ Engl $J$ Med. 2010;363(18):1693-1703. doi:10.1056/NEJMoa1006448

5. Barlesi F, Mazieres J, Merlio J-P, et al. Routine molecular profiling of patients with advanced non-small-cell lung cancer: results of a 1-year nationwide programme of the French cooperative thoracic intergroup (IFCT). Lancet. 2016;387(10026):10026. doi:10.1016/S0140-6736(16)00004-0

6. Gerber DE, Schiller JH. Maintenance chemotherapy for advanced non-small-cell lung cancer: new life for an old idea. Am J Clin Oncol. 2013;31(8):1009-1020. doi:10.1200/JCO.2012.43.7459

7. Hald SM, Rakaee M, Martinez I, et al. LAG-3 in non-small cell lung cancer: expression in primary tumors and metastatic lymph nodes is associated with improved survival. Clin Lung Cancer. 2018:S1525730417303406.

8. Chen BJ, Dashnamoorthy R, Galera P, et al. The immune checkpoint molecules PD-1, PD-L1, TIM-3 and LAG-3 in diffuse large B-cell lymphoma. Oncotarget.2019;10(21):2030

9. Webb ES, Liu P, Baleeiro R, Lemoine NR, Yuan M, Wang Y. Immune checkpoint inhibitors in cancer therapy. $J$ Biomed Res. 2018;32(05):5-14.

10. La-beck NM, Jean GW, Huynh C, et al. Immune checkpoint inhibitors: new insights and current place in cancer therapy. Pharmacotherapy. 2015;35(10):963-976. doi:10.1002/phar.1643

11. Bylicki O, Barazzutti H, Paleiron N, Margery J, Assié JB, Chouaï C. First-line treatment of non-small-cell lung cancer (NSCLC) with immune checkpoint inhibitors. BioDrugs. 2019;33(2):159-71.

12. Dang TO, Ogunniyi A, Barbee MS, Drilon A. Pembrolizumab for the treatment of PD-L1 positive advanced or metastatic non-small cell lung cancer. Expert Rev Anticancer Ther. 2016;16 (1):14737140.2016.1123626.

13. Herbst RS, Baas P, Perez-Gracia JL, et al. Archival Vs New Tumor Samples for Assessing PD-L1 Expression in the KEYNOTE-010 Study of Pembrolizumab (Pembro) Vs Docetaxel (Doce) for Previously Treated Advanced NSCLC. 2016. 
14. Goldberg MV, Maris CH, Hipkiss EL, et al. Role of PD-1 and its ligand, B7-H1, in early fate decisions of CD8 T cells. Blood. 2007;110(1):186-192. doi:10.1182/blood-2006-12-062 422

15. Chemnitz JM, Parry RV, Nichols KE, et al. SHP-1 and SHP-2 associate with immunoreceptor tyrosine-based switch motif of programmed death 1 upon primary human $\mathrm{T}$ cell stimulation, but only receptor ligation prevents $\mathrm{T}$ cell activation. J Immunol. 2004;173(2):945-954. doi:10.4049/jimmunol.173. 2.945

16. Shin $\mathrm{T}$, Yoshimura $\mathrm{K}$, Shin $\mathrm{T}$, et al. In vivo costimulatory role of B7-DC in tuning T helper cell 1 and cytotoxic T lymphocyte responses. J Exp Med. 2005;201(10):1531-1541. doi:10.1084/ jem.20050072

17. Tseng SY, Otsuji M, Gorski K, et al. B7-Dc, a new dendritic cell molecule with potent costimulatory properties for $\mathrm{T}$ cells. J Exp Med. 2001;193(7):839-846. doi:10.1084/ jem.193.7.839

18. Ortler S, Leder C, Mittelbronn M, et al. B7-H1 restricts neuroantigen-specific $\mathrm{T}$ cell responses and confines inflammatory CNS damage: implications for the lesion pathogenesis of multiple sclerosis. Eur J Immunol. 2008;38(6):1734-1744. doi:10.1002/ eji.200738071

19. Latchman Y, Wood CR, Chernova T, et al. PD-L2 is a second ligand for PD-1 and inhibits $\mathrm{T}$ cell activation. Nat Immunol. 2001;2(3):261-268. doi:10.1038/85330

20. Flies DB, Han X, Higuchi T, et al. Coinhibitory receptor PD-1H preferentially suppresses CD4+ T cell-mediated immunity. J Clin Invest. 2014;124(5):1966.

21. Blank C, Brown I, Peterson AC. PD-L1/B7H-1 inhibits the effector phase of tumor rejection by $\mathrm{T}$ cell receptor (TCR) transgenic CD8 + T cells. Cancer Res. 2004;64(3):1140-1145. doi:10.1158/ 0008-5472.CAN-03-3259

22. Dai S, Jia R, Zhang $\mathrm{X}$, et al. The PD-1/PD-Ls pathway and autoimmune diseases. Cell Immunol. 2014;290(1):72-79. doi:10.1016/j.cellimm.2014.05.006

23. Harding CV. Pathways of antigen processing. Curr Opin Immunol. 1991;3(1):3-9. doi:10.1016/0952-7915(91)90068-C

24. Andreotti AH, Schwartzberg PL, Joseph RE, Berg LJ, et al. T-cell signaling regulated by the tec family kinase, itk. Cold Spring Harb Perspect Biol. 2010;2;7:a002287-a002287.

25. Salojin KV, Zhang J, Delovitch TL. TCR and CD28 are coupled via ZAP-70 to the activation of the vav/rac-1-/Pak-1/p38 MAPK signaling pathway. J Immunol. 1999;163(2):844-853.

26. Gasser M. PD-1/PD-L1 expression in colorectal cancer and its implications for apoptosis and tumor immune evasion. Cancer Res. 2006;66(18_suppl):1118.

27. Patsoukis N, Brown J, Petkova V, Liu F, Li L, Boussiotis VA. Selective effects of PD-1 on Akt and ras pathways regulate molecular components of the cell cycle and inhibit $\mathrm{T}$ cell proliferation. Sci Signal. 2012;5;230:ra46-ra46.

28. Kane LP, Weiss A. The PI-3 kinase/Akt pathway and T cell activation: pleiotropic pathways downstream of PIP3. Immunol Rev. 2003;192(1):7-20. doi:10.1034/j.1600-065X.20 03.00008.x

29. Yang Y, Gao M, Lin Z, et al. DEK promoted EMT and angiogenesis through regulating $\mathrm{PI} 3 \mathrm{~K} / \mathrm{AKT} / \mathrm{mTOR}$ pathway in triple-negative breast cancer. Oncotarget. 2017;8(58):98708-98722. doi:10.18632/ oncotarget.21864

30. Hofmeyer KA, Jeon H, Zang X. The PD-1/PD-L1 (B7-H1) Pathway in Chronic Infection-Induced Cytotoxic T Lymphocyte Exhaustion. J Biomed Biotechnol. 2014;2011 (6):451694.

31. Sha SH, Chen F-Q, Schacht J. PTEN attenuates PIP3/Akt signaling in the cochlea of the aging $\mathrm{CBA} / \mathrm{J}$ mouse. Hearing Res. 2010;264(1-2):0-92.
32. De Vos J, Jourdan M, Tarte K, Jasmin C, Klein B. JAK2 tyrosine kinase inhibitor tyrphostin AG490 downregulates the mitogen-activated protein kinase (MAPK) and signal transducer and activator of transcription (STAT) pathways and induces apoptosis in myeloma cells. $\mathrm{Br} J$ Haematol. 2000;109(4):823-828. doi:10.1046/j.1365-2141.2000.02127.x

33. Shie MY, Ding SJ. Integrin binding and MAPK signal pathways in primary cell responses to surface chemistry of calcium silicate cements. Biomaterials. 2013;34(28):6589-6606. doi:10.1016/j. biomaterials.2013.05.075

34. Wartewig T, Kurgyis Z, Keppler S, et al. PD-1 is a haploinsufficient suppressor of $\mathrm{T}$ cell lymphomagenesis. Nature. 2017;552(7683):121-5.

35. He J, Hu Y, Hu M, et al. Development of PD-1/PD-L1 pathway in tumor immune microenvironment and treatment for non-small cell lung cancer. Sci Rep. 2015;5(1):13110. doi:10.1038/srep 13110

36. Rong X, Greening DW, Zhu HJ, Takahashi N, Simpson RJ. Extracellular vesicle isolation and characterization: toward clinical application. J Clin Invest. 2016;126;4:1152-1162.

37. Gang C, Huang AC, Zhang W, et al. Exosomal PD-L1 contributes to immunosuppression and is associated with anti-PD-1 response. Nature. 2018;560(7718):382-6.

38. Hamid O, Robert C, Daud A, et al. Safety and tumor responses with lambrolizumab (anti-PD-1) in melanoma. $N$ Engl $J$ Med. 2013;369(2):134-144. doi:10.1056/NEJMoa1305133

39. Kwok G, Yau TC, Chiu JW, Tse E, Kwong Y. Pembrolizumab (Keytruda). Hum Vaccin. 2016.

40. Robert C, Ribas A, Wolchok JD. Anti-programmed-deathreceptor-1 treatment with pembrolizumab in ipilimumab-refractory advanced melanoma: a randomised dose-comparison cohort of a Phase 1 trial. Lancet. 2014;384 (9948):1109-1117. doi:10.1016/S0140-6736(14)60958-2

41. FDA. Approves merck's KEYTRUDA ${ }^{\circledR}$ (pembrolizumab) for use at an additional recommended dose of $400 \mathrm{mg}$ every six weeks for all approved adult indications. Available from: www.businesswire.com.

42. Gettinger S, Lynch T. A decade of advances in treatment for advanced non-small cell lung cancer. Clin Chest Med. 2011;32 (4):839-851. doi:10.1016/j.ccm.2011.08.017

43. Kelly K, Crowley J, Bunn Jr PA, et al. Randomized phase III trial of paclitaxel plus carboplatin versus vinorelbine plus cisplatin in the treatment of patients with advanced non-small-cell lung cancer: a southwest oncology group trial. J Clin Oncol. 2001;19 (13):3210.

44. Sandler A, Gray RPerry MC, et al. Paclitaxel-Carboplatin Alone or with Bevacizumab for Non-Small-Cell Lung Cancer NEJM. N Engl J Med. 2006.

45. Schiller JH, Harrington D, Belani CP, et al. Comparison of four chemotherapy regimens for advanced non-small-cell lung cancer. N Engl J Med. 2002;346(2):92-98. doi:10.1056/NEJMoa011954

46. Vanmeerbeek I, Sprooten J, De Ruysscher D, et al. Trial watch: chemotherapy-induced immunogenic cell death in immuno-oncology. Oncoimmunology. 2020;9(1):1703449. doi:10. 1080/2162402X.2019.1703449

47. Schaer DA, Geeganage S, Amaladas N, et al. The folate pathway inhibitor pemetrexed pleiotropically enhances effects of cancer immunotherapy. Clin Cancer Res. 2019;25(23):7175. doi:10. 1158/1078-0432.CCR-19-0433

48. Langer CJ, Gadgeel SM, Borghaei $\mathrm{H}$, et al. Carboplatin and pemetrexed with or without pembrolizumab for advanced, non-squamous non-small-cell lung cancer: a randomised, phase 2 cohort of the open-label KEYNOTE-021 study. Lancet Oncol. 2016;17(11):1497-1508. doi:10.1016/S1470-2045(16)30498-3

49. Pembrolizumab (Keytruda) 5-10-2017. Available from: https:// www.fda.gov/Drugs/InformationOnDrugs/ApprovedDrugs/ ucm558048.htm. 
50. Borghaei H, Langer CJ, Gadgeel S, et al. 24-Month overall survival from KEYNOTE-021 cohort G: pemetrexed and carboplatin with or without pembrolizumab as first-line therapy for advanced nonsquamous non-small cell lung cancer. J Thorac Oncol. 2019;14 (1):124-129. doi:10.1016/j.jtho.2018.08.004

51. Gandhi L, Rodríguez-Abreu D, Gadgeel S, et al. Pembrolizumab plus chemotherapy in metastatic non-small-cell lung cancer. $N$ Engl J Med. 2018:NEJMoa1801005.

52. KEYNOTE-189 study of pembrolizumab plus pemetrexed and platinum vs placebo plus pemetrexed and platinum for untreated metastatic nonsquamous NSCLC does choice of platinum affect outcomes. Available from: https://oncologypro.esmo.org/ Meeting-Resources/ESMO-2018-Congress.

53. Notice on Soliciting Opinions on the List of Urgently Needed New Drugs Listed Overseas. Available from: http://www.cde.org $\mathrm{cn} /$ news.do?method=largeInfo\&id=314651.

54. FDA grants regular approval for pembrolizumab in combination with chemotherapy for first-line treatment of metastatic nonsquamous NSCLC. Available from: https://www.fda.gov/Drugs/ InformationOnDrugs/ApprovedDrugs/ucm617471.htm.

55. Xie YL, Liang JZ, Su N. Gefitinib versus erlotinib as first-line treatment for patients with advanced EGFR mutation-positive non-small-cell lung cancer. $J$ South Med Univ. 2015;35 (3):446-449.

56. Kim MH, Shim HS, Kang DR, et al. Clinical and prognostic implications of ALK and ROS1 rearrangements in never-smokers with surgically resected lung adenocarcinoma. Lung Cancer. 2014;83 (3):389-395. doi:10.1016/j.lungcan.2014.01.003

57. Crystal AS, Shaw AT. New targets in advanced NSCLC: EML4-ALK. Clin Adv Hematol Oncol. 2011;9(3):207-214.

58. Heist RS, Mino-Kenudson M, Sequist LV, et al. FGFR1 amplification in squamous cell carcinoma of the lung. $J$ Thorac Oncol. 2012;7(12):1775-1780. doi:10.1097/JTO.0b013e31826aed28

59. Cufer T, Ovcaricek T, O'Brien MER. Systemic therapy of advanced non-small cell lung cancer: major-developments of the last 5-years. Eur J Cancer. 2013;49(6):1216-1225. doi:10.1016/j. ejca.2012.11.021

60. Allemani C, Weir HK, Carreira H. Global surveillance of cancer survival 1995-2009: analysis of individual data for 25,676,887 patients from 279 population-based registries in 67 countries (CONCORD-2). Lancet. 2015;385(9972):977-1010.

61. Reck M, Rodríguez-Abreu D, Robinson AG, et al. Pembrolizumab versus chemotherapy for PD-L1-positive nonsmall-cell lung cancer. $N$ Engl J Med. 2016:NEJMoa1606774.

62. Ettinger DS, Wood DE, Aisner DL. Non-small cell lung cancer, version 5.2017, NCCN clinical practice guidelines in oncology. J Natl Compr Canc Netw. 2017;15(4):504-535. doi:10.6004/ jnccn.2017.0050

63. Paz-Ares L, Luft A, Vicente D, et al. Pembrolizumab plus chemotherapy for squamous non-small-cell lung cancer. $N$ Engl J Med. 2018.

64. Merck's KEYTRUDA ${ }^{\circledR}$ (pembrolizumab) Now approved in China for first-line treatment of metastatic squamous non-small cell lung cancer (NSCLC) in combination with chemotherapy. Available from: https://www.mrknewsroom.com/news-release /oncology-newsroom/mercks-keytruda-pembrolizumab-nowapproved-china-first-line-treatment.

65. Wang DY, Salem J-E, Cohen JV, et al. Fatal toxic effects associated with immune checkpoint inhibitors: a systematic review and meta-analysis. JAMA Oncol. 2018;4(12):1721-1728. doi:10.1001/jamaoncol.2018.3923

66. Mok TS, Wu YL, Kudaba I, et al. Pembrolizumab versus chemotherapy for previously untreated, PD-L1-expressing, locally advanced or metastatic non-small-cell lung cancer (KEYNOTE-042): a randomised, open-label, controlled, Phase 3 trial. Lancet. 2019.
67. Reck M, Rodríguez-Abreu D, Robinson AG, et al. Updated analysis of KEYNOTE-024: pembrolizumab versus platinumbased chemotherapy for advanced non-small-cell lung cancer with PD-L1 tumor proportion score of $50 \%$ or greater. J Clin Oncol. 2019;37(7):537-546. doi:10.1200/JCO.18.00149

68. Pembrolizumab (KEYTRUDA) checkpoint inhibitor. Available from: https://www.fda.gov/drugs/resources-information-approved -drugs/pembrolizumab-keytruda-checkpoint-inhibitor.

69. Pilkington G, Boland A, Brown T, et al. A systematic review of the clinical effectiveness of first-line chemotherapy for adult patients with locally advanced or metastatic non-small cell lung cancer. Thorax. 2015;70(4):359. doi:10.1136/thoraxjnl-2014-205914

70. Killock D. Lung cancer: frontline nivolumab - checkmate 026 ends in stalemate. Nat Rev Clin Oncol. 2017;14(8):458. doi:10.1038/nrclinonc.2017.102

71. Hui R, Garon EB, Goldman JW, et al. Pembrolizumab as first-line therapy for patients with PD-L1-positive advanced non-small cell lung cancer: a phase 1 trial. Ann Oncol. 2017;28(4):874-881. doi:10.1093/annonc/mdx008

72. Dietel M, Savelov N, Salanova R, et al. Real-world prevalence of programmed death ligand 1 expression in locally advanced or metastatic non-small-cell lung cancer: the global, multicenter EXPRESS study. Lung Cancer. 2019;134:174-179. doi:10.1016/ j.lungcan.2019.06.012

73. FDA Expands pembrolizumab indication for first-line treatment of NSCLC (TPS $\geq 1 \%$ ). Available from: https://www.fda.gov/ drugs/fda-expands-pembrolizumab-indication-first-line-treatmentnsclc-tps-1.

74. Wang Y, Zhou S, Yang F, et al. Treatment-related adverse events of PD-1 and PD-L1 inhibitors in clinical trials: a systematic review and meta-analysis. JAMA. 2019;5.

75. Abou Alaiwi S, Xie W, Nassar AH, et al. Safety and efficacy of restarting immune checkpoint inhibitors after clinically significant immune-related adverse events in metastatic renal cell carcinoma. J Immunother Cancer. 2020;8(1):e000144. doi:10.1136/jitc-2019000144

76. Baxi S, Yang A, Gennarelli RL, et al. Immune-related adverse events for anti-PD-1 and anti-PD-L1 drugs: systematic review and meta-analysis. BMJ Clin Res. 2018;360:k793. doi:10.1136/bmj.k793

77. Hofmann L, Forschner A, Loquai C. Cutaneous, gastrointestinal, hepatic, endocrine, and renal side-effects of anti-PD-1 therapy. Eur J Cancer. 2016.

78. Hamid O, Robert C, Daud A, et al. Five-year survival outcomes for patients with advanced melanoma treated with pembrolizumab in KEYNOTE-001. Ann Oncol. 2019;30(4):582-588. doi:10. 1093/annonc/mdz011

79. Topalian SL, Sznol M, McDermott DF. Survival, durable tumor remission, and long-term safety in patients with advanced melanoma receiving nivolumab. J Clin Oncol. 2014;32 (10):1020-1030. doi:10.1200/JCO.2013.53.0105.

80. Sibaud V, David I, Lamant L, et al. Acute skin reaction suggestive of pembrolizumab-induced radiosensitization. Melanoma Res. 2015;25(6):555. doi:10.1097/CMR.0000000000000191

81. Sanlorenzo M, Vujic I, Daud A, et al. Pembrolizumab cutaneous adverse events and their association with disease progression. JAMA Dermatol. 2015;151(11):1206-1212. doi:10.1001/jamadermatol.2015.1916

82. Weber JS, Kahler KC, Hauschild A. Management of immune-related adverse events and kinetics of response with ipilimumab. J Clin Oncol. 2012;30(21):2691-2697. doi:10.1200/ JCO.2012.41.6750

83. Shaverdian N, Lisberg AE, Bornazyan K, et al. Previous radiotherapy and the clinical activity and toxicity of pembrolizumab in the treatment of non-small-cell lung cancer: a secondary analysis of the KEYNOTE-001 phase 1 trial. Lancet Oncol. 2017;18 (7):895-903. doi:10.1016/S1470-2045(17)30380-7 
84. Kähler KC, Hassel JC, Heinzerling L, et al. Management of side effects of immune checkpoint blockade by anti-CTLA-4 and anti-PD-1 antibodies in metastatic melanoma: CME article. $J$ Dtsch Dermatol Ges. 2016;14(7):662-681.

85. Michot JM, Bigenwald C, Champiat $\mathrm{S}$, et al. Immune-related adverse events with immune checkpoint blockade: a comprehensive review. Eur J Cancer. 2016;54:139-148. doi:10.1016/j.ejca.2015.11.016

86. Herbst RS, Baas P, Kim DW, et al. Pembrolizumab versus docetaxel for previously treated, PD-L1-positive, advanced non-small-cell lung cancer (KEYNOTE-010): a randomised controlled trial. Lancet. 2016;387(10027):1540-1550.

87. Johncilla M, Misdraji J, Pratt DS, et al. Ipilimumab-associated hepatitis: clinicopathologic characterization in a series of 11 cases. Am J Surg Pathol. 2015;39(8):1075. doi:10.1097/ PAS. 0000000000000453

88. Kim KW, Ramaiya NH, Krajewski KM, et al. Ipilimumab associated hepatitis: imaging and clinicopathologic findings. Invest New Drugs. 2013;31(4):1071-1077. doi:10.1007/s10637-0139939-6

89. Nishino M, Sholl LM, Hatabu H, et al. Anti-PD-1-related pneumonitis during cancer immunotherapy. $N$ Engl J Med. 2015;373 (3):288-290. doi:10.1056/NEJMc1505197

90. Nishino M, Giobbie-Hurder A, Hatabu $\mathrm{H}$, et al. Incidence of programmed cell death 1 inhibitor-related pneumonitis in patients with advanced cancer: a systematic review and meta-analysis. JAMA Oncol. 2016;2(12):1607.

91. Kang SP, Gergich K, Lubiniecki GM, et al. Pembrolizumab KEYNOTE-001: an adaptive study leading to accelerated approval for two indications and a companion diagnostic. Ann Oncol. 2017;28(6):1388-1398. doi:10.1093/annonc/mdx 076

92. Gulley JL, Rajan A, Spigel DR, et al. Avelumab for patients with previously treated metastatic or recurrent non-small-cell lung cancer (JAVELIN solid tumor): dose-expansion cohort of a multicentre, open-label, phase 1b trial. Lancet Oncol. 2017: S1470204517302401.

93. Nishino M, Brais LK, Brooks NV, et al. Drug-related pneumonitis during mammalian target of rapamycin inhibitor therapy in patients with neuroendocrine tumors: a radiographic pattern-based approach. Eur $J$ Cancer. 2016;53:163-170.

94. Eigentler TK, Hassel JC, Berking C, et al. Diagnosis, monitoring and management of immune-related adverse drug reactions of anti-PD-1 antibody therapy. Cancer Treat Rev. 2016;45:7-18. doi:10.1016/j.ctrv.2016.02.003

95. Torino F, Barnabei A, Paragliola R, et al. Endocrine side-effects of anti-cancer drugs: mAbs and pituitary dysfunction: clinical evidence and pathogenic hypotheses. Eur $J$ Endocrinol. 2013;169(6):153-164.

96. Ryder M, Callahan M, Postow MA, et al. Endocrine-related adverse events following ipilimumab in patients with advanced melanoma: a comprehensive retrospective review from a single institution. Endocr Relat Cancer. 2014;21(2):371-381. doi:10. 1530/ERC-13-0499

97. Mellati M, Eaton KD, Brooks-Worrell BM, et al. Anti-PD-1 and anti-PDL-1 monoclonal antibodies causing type 1 diabetes. Diabetes Care. 2015;38(9):e137-e138. doi:10.2337/dc15-0889

98. Spain L, Diem S, Larkin J. Management of toxicities of immune checkpoint inhibitors. Cancer Treat Rev. 2016:S030573721 6000165 .

99. Pagès C, Gornet JM, Monsel G, et al. Ipilimumab-induced acute severe colitis treated by infliximab. Melanoma Res. 2013;23 (3):227-230. doi:10.1097/CMR.0b013e32835fb524

100. Nishino M, Chambers ES, Chong CR, et al. Anti-PD-1 inhibitor-related pneumonitis in non-small cell lung cancer. Cancer Immunol Res. 2016:2326-6066.CIR-15-0267.
101. Naidoo J, Page DB, Li BT, et al. Toxicities of the anti-PD-1 and anti-PD-L1 immune checkpoint antibodies. Ann Oncol. 2015: mdv383.

102. Ott PA, Elez E, Hiret S, et al. Pembrolizumab in patients with extensive-stage small-cell lung cancer: results from the phase $\mathrm{Ib}$ KEYNOTE-028 study. Am J Clin Oncol. 2017;35 (34):3823-3829. doi:10.1200/JCO.2017.72.5069

103. Hsu C, Lee S-H, Ejadi S, et al. Safety and antitumor activity of pembrolizumab in patients with programmed death-ligand 1positive nasopharyngeal carcinoma: results of the KEYNOTE028 study. Am J Clin Oncol. 2017;35(36):JCO2017733675. doi:10.1200/JCO.2017.73.3675

104. Blank C, Gajewski TF, Mackensen A. Interaction of PD-L1 on tumor cells with PD-1 on tumor-specific T cells as a mechanism of immune evasion: implications for tumor immunotherapy. Cancer Immunol Immunother. 2005;54(4):307-314. doi:10.1007/ s00262-004-0593-x

105. Patel SP, Kurzrock R. PD-L1 expression as a predictive biomarker in cancer immunotherapy. Mol Cancer Ther. 2015;14 (4):847-856. doi:10.1158/1535-7163.MCT-14-0983

106. Vokes EE, Ready N, Felip E, et al. Nivolumab versus docetaxel in previously treated advanced non-small cell lung cancer (CheckMate 017 and CheckMate 057): 3-year update and outcomes in patients with liver metastases. Ann Oncol. 2018.

107. Mazieres J, Kowalski D, Luft A, et al. Health-related quality of life with carboplatin-paclitaxel or nab-paclitaxel with or without pembrolizumab in patients with metastatic squamous non-smallcell lung cancer. J Clin Oncol. 2020;38(3):271-280. doi:10.1200/ JCO.19.01348

108. Garassino MC, Gadgeel S, Esteban E, et al. Patient-reported outcomes following pembrolizumab or placebo plus pemetrexed and platinum in patients with previously untreated, metastatic, non-squamous non-small-cell lung cancer (KEYNOTE-189): a multicentre, double-blind, randomised, placebo-controlled, phase 3 trial. Lancet Oncol. 2020.

109. Chae YK, Pan A, Davis AA, et al. Biomarkers for PD-1/PD-L1 blockade therapy in non-small-cell lung cancer: is PD-L1 expression a good marker for patient selection? Clin Lung Cancer. 2016;17(5):350-361. doi:10.1016/j.cllc.2016.03.011

110. Xu F, Xu L, Wang Q, et al. Clinicopathological and prognostic value of programmed death ligand-1 (PD-L1) in renal cell carcinoma: a meta-analysis. Int J Clin Exp Med. 2015;8(9):14595-14603.

111. Janjigian YY, Bendell JC, Calvo E, et al. Checkmate-032: Phase I/II, Open-Label Study of Safety and Activity of Nivolumab (Nivo) Alone or with Ipilimumab (Ipi) in Advanced and Metastatic (A/M) Gastric Cancer (GC). 2016.

112. Rosenberg JE, Hoffman-Censits J, Powles T, et al. Atezolizumab in patients with locally advanced and metastatic urothelial carcinoma who have progressed following treatment with platinum-based chemotherapy: a single-arm, multicentre, phase 2 trial. Lancet. 2016;S0140673616005614.

113. Le DT, Durham JN, Smith KN, et al. Mismatch repair deficiency predicts response of solid tumors to PD-1 blockade. Science. 2017;357(6349):409-413. doi:10.1126/science.aan6733

114. Rizvi NA, Hellmann MD, Snyder A, et al. Mutational landscape determines sensitivity to PD-1 blockade in non-small cell lung cancer. Science. 2015;348(6230):124-128. doi:10.1126/science.aaa1348

115. Anagnostou V, Smith KN, Forde PM, et al. Evolution of neoantigen landscape during immune checkpoint blockade in non-small cell lung cancer. Cancer Discov. 2017;7(3):264-276. doi:10.1158/ 2159-8290.CD-16-0828

116. Rosenberg JE, Hoffman-Censits J, Powles T, et al. Atezolizumab in patients with locally advanced and metastatic urothelial carcinoma who have progressed following treatment with platinum-based chemotherapy: a single-arm, multicentre, phase 2 trial. Lancet. 2016;387 (10031):1909-1920. doi:10.1016/S0140-6736(16)00561-4 
117. Iyer RR, Pluciennik A, Burdett V, et al. DNA mismatch repair: functions and mechanisms. Chem Rev. 2006;106(2):302-323. doi:10.1021/cr0404794

118. Kelderman S, Schumacher TN, Kvistborg P. Mismatch repair-deficient cancers are targets for anti-PD-1 therapy. Cancer Cell. 2015;28(1):11-13. doi:10.1016/j.ccell.2015.06.012

119. Li Q, Zhang B, Niu FN, et al. Clinicopathological characteristics, MSI and K-ras gene mutations of double primary malignancies associated with colorectal cancer. Zhonghua Yi Xue Za Zhi. 2020;100 (4):301-306. doi:10.3760/cma.j.issn.0376-2491.2020.04.012

120. Bupathi $\mathrm{M}, \mathrm{Wu} \mathrm{C}$. Biomarkers for immune therapy in colorectal cancer: mismatch-repair deficiency and others. $J$ Gastrointest Oncol. 2016;7(5):713-720. doi:10.21037/jgo.2016.07.03

121. Release FN. FDA Approves First Cancer Treatment for Any Solid Tumor with a Specific Genetic Feature. 2017.

122. Le DT, Uram JN, Wang H, et al. PD-1 blockade in tumors with mismatch-repair deficiency. $N$ Engl $J$ Med. 2015;372 (26):2509-2520. doi:10.1056/NEJMoa1500596

123. Lisberg A, Cummings A, Goldman JW, et al. A phase ii study of pembrolizumab in EGFR-mutant, PD-L1+, tyrosine kinase inhibitor naive patients with advanced NSCLC. $J$ Thorac Oncol. 2018;13(8):1138-1145. doi:10.1016/j.jtho.2018.03.035

124. Champiat S, Dercle L, Ammari S, et al. Hyperprogressive disease is a new pattern of progression in cancer patients treated by anti-PD-1/PD-L1. Clin Cancer Res. 2017;23(8):1920-1928. doi:10.1158/1078-0432.CCR-16-1741

125. Kato S, Goodman A, Walavalkar V, et al. Hyperprogressors after immunotherapy: analysis of genomic alterations associated with accelerated growth rate. Clin Cancer Res. 2017;23 (15):4242-4250. doi:10.1158/1078-0432.CCR-16-3133
126. Sanmamed MF, Chen L. A paradigm shift in cancer immunotherapy: from enhancement to normalization. Cell. 2019;176(3):677. doi:10.1016/j.cell.2019.01.008

127. Lommatzsch M, Bratke K, Stoll P. Neoadjuvant PD-1 blockade in resectable lung cancer. $N$ Engl J Med. 2018;379(9):e14.

128. Waks AG, Winer EP. Breast cancer treatment: a review. JAMA. 2019;321(3):288-300. doi:10.1001/jama.2018.19323

129. Meng X, Huang Z, Teng F, et al. Predictive biomarkers in PD-1/ PD-L1 checkpoint blockade immunotherapy. Cancer Treat Rev. 2015;41(10):868-876. doi:10.1016/j.ctrv.2015.11.001

130. Dudley JC, Lin M-T, Le DT, et al. Microsatellite instability as a biomarker for PD-1 blockade. Clin Cancer Res. 2016;22 (4):813-820. doi:10.1158/1078-0432.CCR-15-1678

131. Lim C, Tsao MS, Le LW, et al. Biomarker testing and time to treatment decision in patients with advanced nonsmall-cell lung cancer. Ann Oncol. 2015;26(7):1415-1421. doi:10.1093/annonc/ mdv208

132. Huang Y, Yuan J, Righi E, et al. Vascular normalizing doses of antiangiogenic treatment reprogram the immunosuppressive tumor microenvironment and enhance immunotherapy. Proc Natl Acad Sci U S A. 2012;109(43):17561-17566. doi:10.1073/ pnas. 1215397109

133. Motzer RJ, Rini BI, McDermott DF, et al. Nivolumab plus ipilimumab versus sunitinib in first-line treatment for advanced renal cell carcinoma: extended follow-up of efficacy and safety results from a randomised, controlled, phase 3 trial. Lancet Oncol. 2019;20(10):1370-1385. doi:10.1016/S1470-2045(19)30413-9

\section{Publish your work in this journal}

Cancer Management and Research is an international, peer-reviewed open access journal focusing on cancer research and the optimal use of preventative and integrated treatment interventions to achieve improved outcomes, enhanced survival and quality of life for the cancer patient.
The manuscript management system is completely online and includes a very quick and fair peer-review system, which is all easy to use. Visit http://www.dovepress.com/testimonials.php to read real quotes from published authors. 